\title{
Influence of longitudinally tapered collimators on a high brightness electron beam
}

\author{
S. Di Mitri, L. Froehlich, and E. Karantzoulis \\ Sincrotrone Trieste, 34149 Basovizza, Trieste, Italy \\ (Received 23 March 2012; published 13 June 2012)
}

\begin{abstract}
This article presents the design and operation of a longitudinally tapered collimator in a single-pass $S$-band linac driving a high brightness electron beam. Measurements were done for the transverse emittance growth induced by the collimator wakefield as a function of the lateral displacement of the beam inside the collimator and the energy acceptance provided by an identical collimator installed in a dispersive region. The measurements demonstrate that: (i) the proposed design allows very precise and reproducible motion down to the micron level of the compact, four-hole collimator; (ii) the collimator does not degrade the beam emittance in the presence of standard trajectory control; (iii) the measured kick factor and energy acceptance are in agreement with the theoretical expectations. These measurements were made using 500 pC, 2.4 ps long bunches at the FERMI@Elettra free electron laser facility.
\end{abstract}

\section{INTRODUCTION}

In high-energy linear colliders, collimators are generally metallic blocks that can be used to protect detectors from being directly struck by a beam pulse that is not properly controlled in optics or energy and to minimize the detector background from halo particles while transmitting core particles to the collision point. In linac-driven light sources, collimators prevent halo particles from hitting the vacuum chamber and then creating electromagnetic showers that can destroy electronics and/or demagnetize permanent magnet blocks in undulators. In all these cases, the collimators are designed to restrict the vacuum chamber physical aperture without affecting the main beam. Usually, the collimator aperture is of the order of few millimeters to few centimeters. Depending on the bunch charge, the bunch length, and the collimator geometry, a small aperture could generate significant transverse wakefields that impart a kick to the beam. This kick strength is correlated with the particle longitudinal coordinate. As a consequence, the collimator wakefield can reduce luminosity or brilliance through trajectory jitter amplification (by a net centroid deflection) and increase the projected emittance (by the head-tail lateral displacement).

This article examines the single-pass effect of a longitudinally tapered collimator upon an ultrarelativistic, high brightness electron beam. In the archival literature, several studies can be found about the collimation system design for linacs [1-4] and the computation of collimator wake functions [5-10]. At least four works [11-14] report direct measurements of the kick factor of tapered collimators with the purpose of benchmarking analytical models of

Published by the American Physical Society under the terms of the Creative Commons Attribution 3.0 License. Further distribution of this work must maintain attribution to the author(s) and the published article's title, journal citation, and DOI. the wakefield. The kick factor was measured by determining the beam deflection angle induced by the collimator as a function of the beam lateral displacement. These studies, however, did not examine the collimator wakefield effects upon the beam emittance nor the energy acceptance of the line. Furthermore, to date no studies of beam quality during the routine machine operation in the presence of collimation have been published since the advent of electron linacs driven by high brightness photoinjectors; these linacs typically provide a transverse normalized emittance equal to or smaller than $1 \mathrm{~mm}$ mrad and are therefore very sensitive to any small perturbation during the beam transport.

The remainder of this article is organized as follows: Section II discusses the engineering design of the tapered collimator $[15,16]$ for the FERMI@Elettra 1.2 to $1.8 \mathrm{GeV}$ linac-driven free electron laser (FEL) [17]. Identical collimators are used in this facility for both geometric and energy collimation. Section III summarizes the electron optics design in the collimators area. Section IV presents experimental results at the FERMI facility in which the transverse emittance is measured downstream of a geometric collimator as a function of the beam lateral position inside the collimator. The collimator kick factor is thus evaluated and compared with the theoretical estimate. Section $V$ shows the measurement of the energy acceptance as determined by a collimator placed in the dispersive region of the FERMI high-energy transfer line. This experiment consisted of steering the trajectory inside the collimator and of measuring the charge transmitted to the end of the line. A reasonable agreement was found between the experimental results and the predictions from the theoretical model. We close with our conclusions in Sec. VI.

\section{COLLIMATOR DESIGN}

Collimators are usually composed of two parts, the spoiler and the absorber. The thickness of the absorber is 

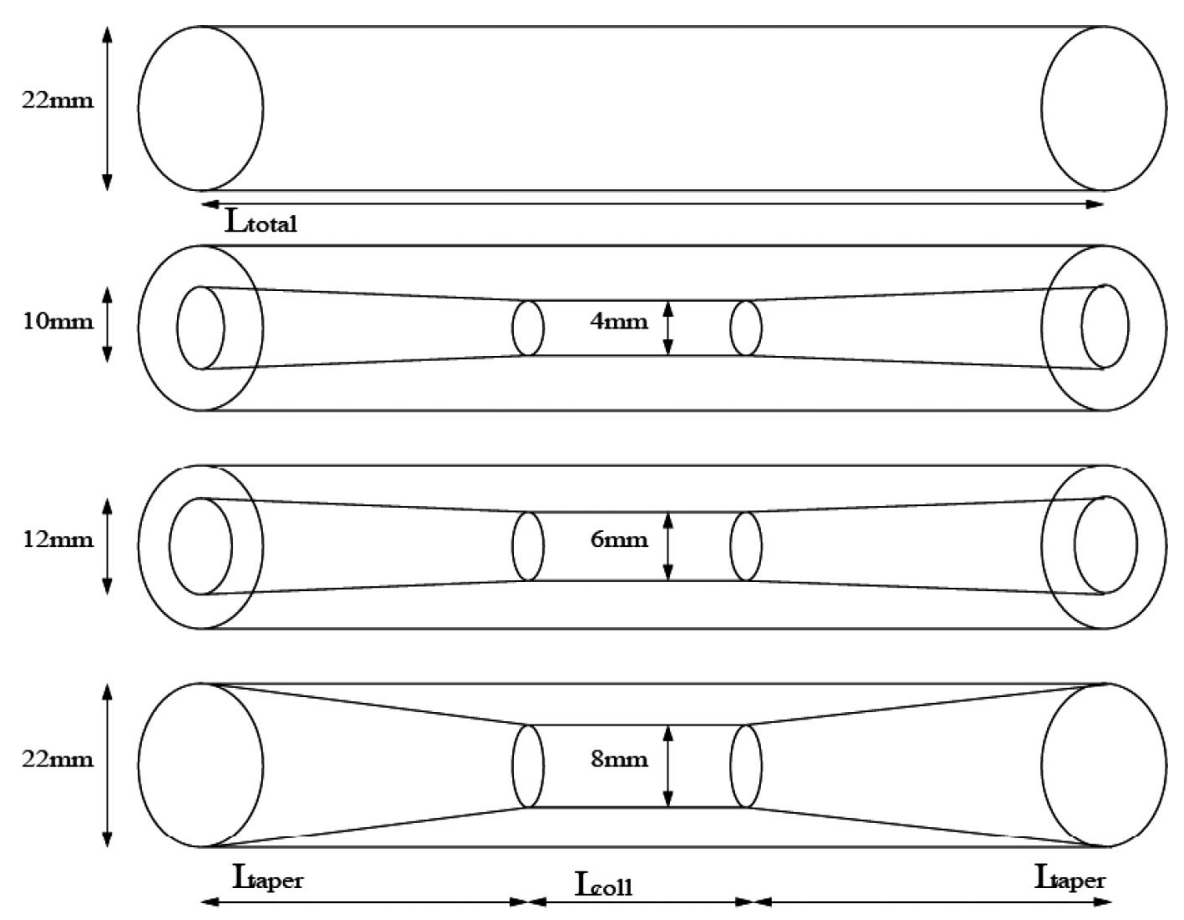

FIG. 1. Four holes FERMI collimator design. $L_{\text {total }}=455 \mathrm{~mm}, L_{\text {taper }}=185 \mathrm{~mm}, L_{\text {coll }}=85 \mathrm{~mm}$.

normally about 10 radiation lengths, while that of the spoiler is $2-3$ radiation lengths. Ideally, two collimation systems (spoiler plus absorber) should be separated by $\pi / 2$ betatron phase advance in order to maximize the interception of particles in the transverse phase space. Unfortunately, this scheme is quite space consuming. In order to minimize the longitudinal space required for the collimators, cylindrical fixed apertures were chosen for FERMI, thus both planes are taken care of simultaneously. Moreover, the spoiler plus absorber scheme was abandoned in favor of one single compact metallic block $[15,16]$.

To minimize the effect of wakefields, the aperture profiles are tapered in a similar fashion to the ones sketched in [10]. However, the final design was constrained by the space available for these devices in the predetermined FERMI layout. To allow flexibility in the beam optics, four apertures with longitudinal tapering are allocated in the same block. The apertures have been machined on the same vertical axis with micron accuracy. This allows only one degree of freedom when changing holes with obvious operational advantages such as the speed and the alignment accuracy. Figure 1 shows the four different apertures: no collimation (the hole is as large as the vacuum chamber), defined collimation (step-in at $10 \mathrm{~mm}$ diameter followed by tapers with an inclination of $1.5^{\circ}$ down to the $4 \mathrm{~mm}$ diameter hole), and two intermediate collimations.

Starting from the shape of Fig. 1 for the internal profile, the external dimensions were scaled in length and thickness while evaluating with particle tracking the percentage of absorbed energy, stopped electrons, escaping particles, and temperature rise. Impedance simulations were performed to define the taper size. The simulation studies are reported in $[15,16]$. The final design shows a predicted stopping efficiency higher than $90 \%$ in the case of a $1.5 \mathrm{GeV}$ and $1 / r$ primary halo electron radial distribution. At FERMI's design repetition rate of $50 \mathrm{~Hz}$, the interception of $10 \mathrm{pC}$ per bunch would cause only a negligible temperature rise. In the worst case of a mis-steered $1 \mathrm{nC}$ beam continuously impinging on the collimator in the full absence of water cooling, the temperature rise could reach $75^{\circ} \mathrm{C}$ in a few

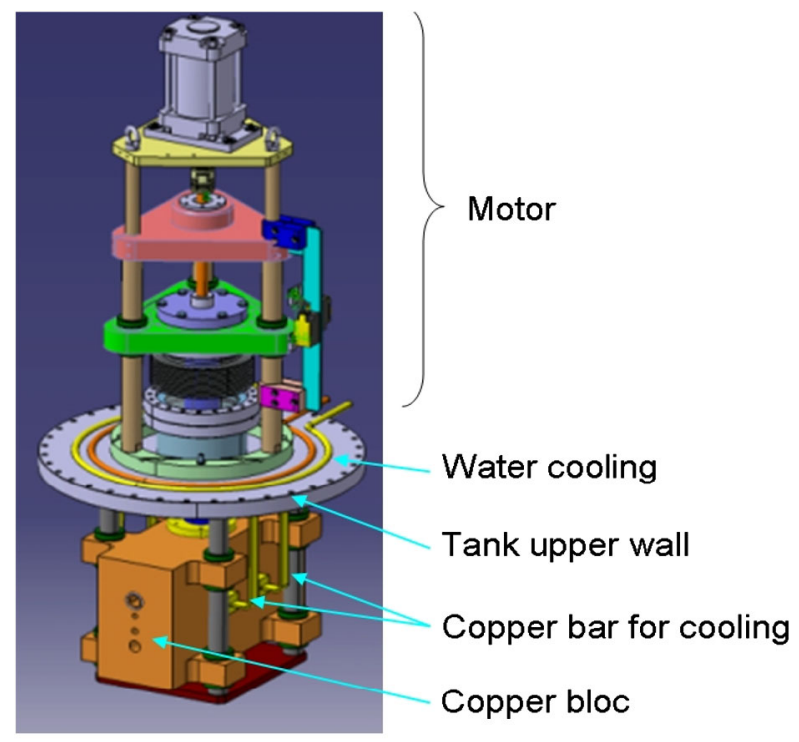

FIG. 2. Engineering design of the FERMI collimator. 
seconds. Copper has been chosen for the collimator material as a compromise between the (moderate) susceptibility to activation and the (short) radiation length, $L_{C u}=$ $14 \mathrm{~mm}$. For this choice, the collimator could survive without damage at temperatures as high as $200^{\circ} \mathrm{C}$. The copper block is positioned on a Phytron stepper motor controlled via a Gefran linear encoder. Allowable vertical movement of the block in the range $0-90 \mathrm{~mm}$ is achieved with $5 \mu \mathrm{m}$ steps and $1 \mu \mathrm{m}$ reproducibility. A 3D view of the collimator system is shown in Fig. 2.

\section{OPTICS DESIGN}

The design optics in the geometric collimation area is a symmetric low-beta, dispersion-free insertion. Two collimators are placed at the edges where $\beta_{x}=\beta_{y}=11 \mathrm{~m}$. Given other optics constraints due to the available space and required diagnostics setup [18], the relative phase advance between two consecutive collimators was set to $2 \pi / 3$. The resultant collimation efficiency is somewhat reduced when compared to the ideal $\pi / 2$ case. Equation (11) in [19] can be used to evaluate the geometric collimation efficiency of the FERMI system compared to the ideal case; this being defined both in terms of the optimum collimator gap and the optimum phase advance. Our calculations showed an efficiency of $76 \%$ for protecting the $3.5 \mathrm{~mm}$ half-gap undulator vacuum chamber from particle impact with a safety margin of $10 \%$. Here, the collimator half-gap $g=2 \mathrm{~mm}$, the betatron function at the collimator $\beta=11 \mathrm{~m}$, the maximum $\beta$ in the undulator $=$ $10 \mathrm{~m}$, and the phase advance was $2 \pi / 3$. In order to compensate for this imperfect efficiency, we adopted a redundant geometric collimation scheme for FERMI with the first collimation system placed at the energy of $320 \mathrm{MeV}$ (right past the first magnetic compressor) and the second one at the linac end.

\section{EMITTANCE MEASUREMENT}

We made transverse emittance measurements of a $500 \mathrm{pC}, 2.4 \mathrm{ps}$ rms long electron bunch via the quadrupole scan technique $[20,21]$ downstream of the first collimator, in the first compressor area, at the mean energy of
321.9 MeV. A sketch of the diagnostic line is given in Fig. 3.

Initially, the collimator was set to its larger hole. The beam was optically matched to the design lattice with a mismatch parameter [22] equal to 1.1. A normalized rms projected emittance of $0.9 \mathrm{~mm}$ mrad was measured in both planes. The rms beam size measured with a yttrium aluminum garnet (YAG) scintillating screen placed $1 \mathrm{~m}$ downstream of the collimator was $\sigma=140 \mu \mathrm{m}$, in good agreement with the model for a Gaussian beam. For the case of the $2 \mathrm{~mm}$ radius hole, the inner wall of the collimator has a distance of approximately $15 \sigma$ from the beam. The beam typically looks compact in the configuration space and it is not intercepted by the collimator, even at the smallest gap. The beam trajectory was controlled by a feedback system utilizing strip-line beam position monitors (BPMs) with $5 \mu \mathrm{m}$ resolution. The beam was initially centered on the BPM that is $1.1 \mathrm{~m}$ upstream of the collimator. By measuring the beam position at the two screens downstream of the collimator, we determined a centroid angular divergence of $\left(x^{\prime}, y^{\prime}\right)=(200 \mu \mathrm{rad}, 80 \mu \mathrm{rad}) \pm$ $50 \mu \mathrm{rad}$. For the given layout, this implies a beam position offset of the order of $1 \sigma$ in the collimator when the beam is centered in the BPM.

Next, the collimator was moved to the $2 \mathrm{~mm}$ radius hole. The transverse projected emittance was measured as a function of various trajectory offsets in order to monitor the effect of the collimator transverse wakefield. Note that, in this experiment, the resistive-wall wakefield is orders of magnitude weaker than the geometric wake and will therefore not be considered in this paper. The experiment was carried out only for the smallest collimator hole because, as mentioned above, that hole is the only one that ensures a safe undulator protection when using the routine optics for the FERMI operation. Clearly, this hole is expected to pose the most critical risk when considering preservation of the beam transverse emittance.

The predicted emittance growth induced by the collimator geometric wakefield can be evaluated by means of the beam matrix formalism, together assuming a Gaussian particle distribution in the phase space and a single-kick perturbation. The wake kick is [14]

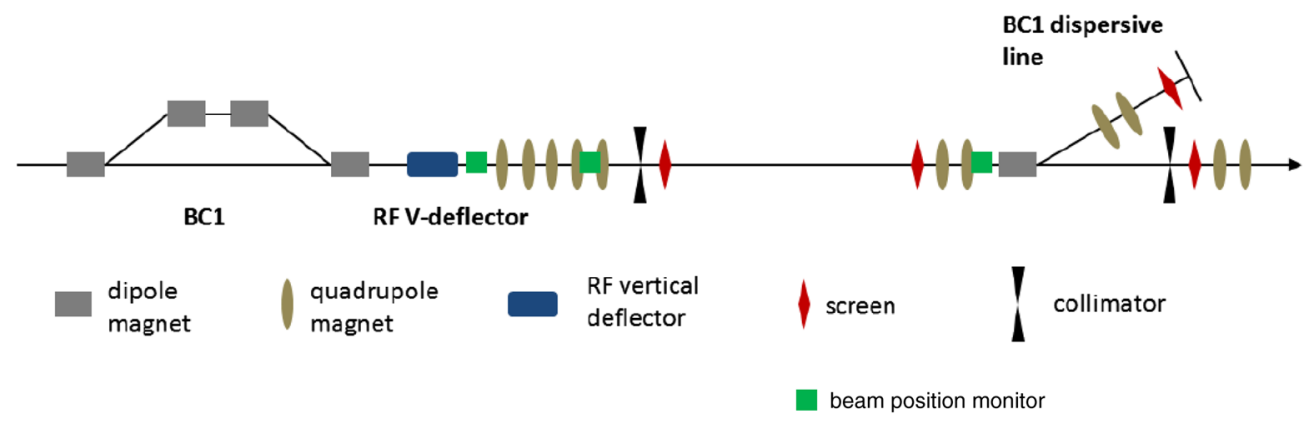

FIG. 3. FERMI geometric collimation insertion after the first magnetic compressor (not to scale). 


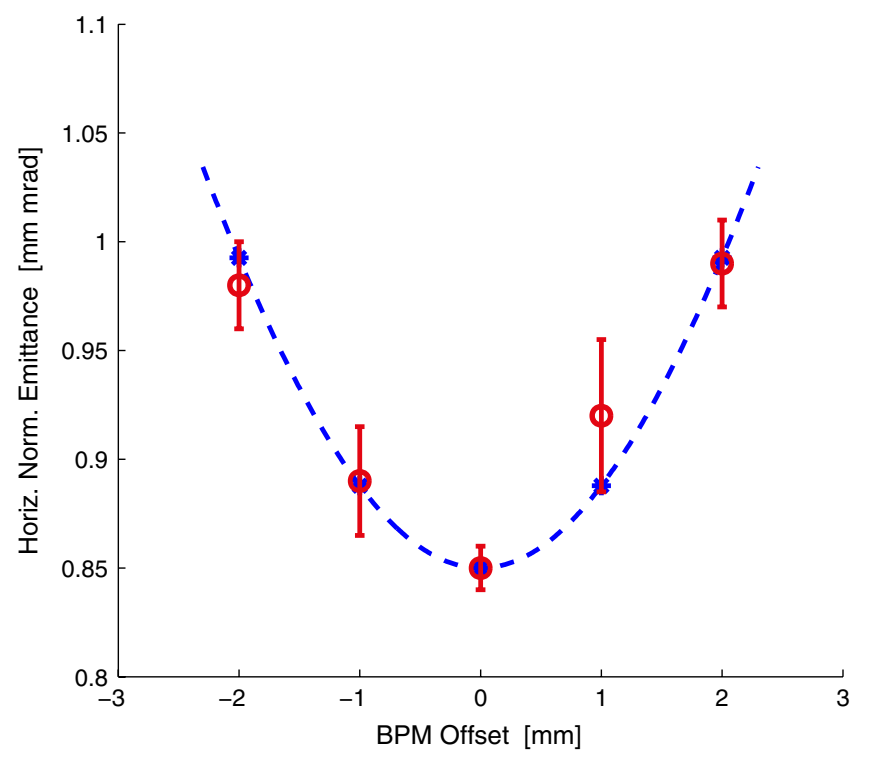

FIG. 4. The horizontal normalized emittance vs the BPM horizontal offset. The geometric collimator is set to the $2 \mathrm{~mm}$ half-gap hole. The error bars show the maximum uncertainty of four consecutive emittance measurements. The quadratic term of the fitting corresponds to a kick factor of $\kappa_{\text {fit }}=2.20 \mathrm{~V} / \mathrm{pC} / \mathrm{mm}$. The dashed curve shows Eq. (2) evaluated for $\kappa=\kappa_{\text {fit }}$.

$$
\theta=\frac{h Q \kappa}{E}
$$

where $Q$ is the bunch charge, $h$ is the bunch centroid distance from the collimator axis, $\kappa$ is the kick factor in the plane of interest - the transverse kick averaged over the length of the beam, and $E$ is the beam mean energy. The normalized emittance growth in the horizontal plane is computed from the determinant of the perturbed beam matrix:

$$
\gamma \varepsilon_{x}=\gamma \sqrt{\varepsilon_{x, 0}^{2}\left(1+\frac{\beta_{x}}{\varepsilon_{x, 0}} \theta^{2}\right)}
$$

where $\varepsilon_{x, 0}$ is the unperturbed rms geometric emittance and $\gamma$ is the Lorentz factor.

Figure 4 shows the measured emittance growth as a function of the beam offset at the BPM. The emittance error is determined by the measurement reproducibility. From a polynomial fitting of the measured data, the effective kick factor that characterizes the FERMI collimator is computed: $\kappa_{\mathrm{fit}}=2.20 \mathrm{~V} / \mathrm{pC} / \mathrm{mm}$.

In spite of the relatively long taper, the FERMI collimator set to a $2 \mathrm{~mm}$ radius hole has a significant step-in and step-out whose thickness is $6 \mathrm{~mm}$ and thus comparable to the collimator outer radius (see Fig. 1). The effective taper angle at the entrance and at the exit of the collimator is therefore bigger than 1 and the wakefield should be modeled in the diffractive regime. Given the flat region at the minimum aperture is a small fraction of the total length, we estimate the kick factor adopting the short collimator approximation [see [14], Eq. (7)] and obtain $\kappa_{D}=$ $2.19 \mathrm{~V} / \mathrm{pC} / \mathrm{mm}$. We notice that a similar taper design, named "Set 1", was independently studied for the FLASH facility in Ref. [10]. The maximum transverse wake potentials computed for the FLASH and the FERMI collimator are $3 \mathrm{~V} / \mathrm{pC} / \mathrm{mm}$ [10] and $4 \mathrm{~V} / \mathrm{pC} / \mathrm{mm}$ [16], respectively. The kick factor evaluated in the FLASH case was $\kappa=2.04 \mathrm{~V} / \mathrm{pC} / \mathrm{mm}$, a value close to our experimental and theoretical findings.

\section{ENERGY ACCEPTANCE MEASUREMENT}

The energy acceptance of the FERMI collimation system was determined by measuring the charge transmitted through the $30 \mathrm{~m}$ long transfer line as a function of the beam trajectory offset in the collimator. The lateral position of the beam is actually measured at a BPM located $0.65 \mathrm{~m}$ upstream of the collimator. The rms beam transverse size measured at a YAG screen $1.2 \mathrm{~m}$ downstream of the collimator is $149 \mu \mathrm{m}$ in the horizontal plane and $64 \mu \mathrm{m}$ in the vertical one, respectively.

The energy acceptance is defined in terms of the beam offset $\Delta x_{T}$ at which the charge starts decreasing, i.e., where the beam starts being intercepted by the collimator:

$$
\frac{\Delta E}{E}=\frac{\Delta x_{T}}{\eta_{x}},
$$

where $\eta_{x}$ is the horizontal dispersion at the collimator. The dispersion was measured by changing the beam mean energy over a normalized range of $\pm 1.5 \%$ (by varying the electric field amplitude in the last accelerating structure

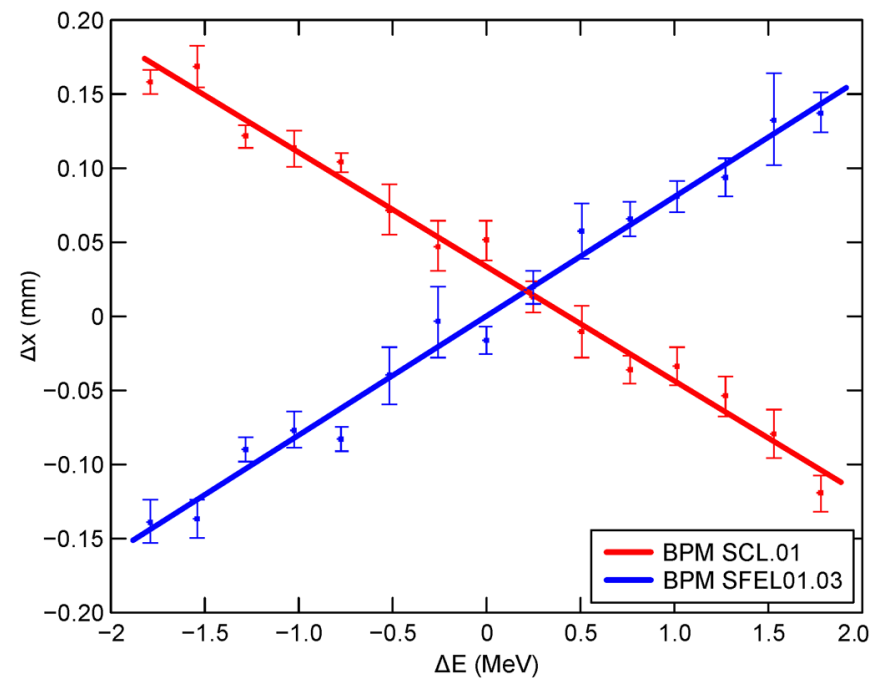

FIG. 5. The horizontal dispersion measurement at two BPMs in the FERMI high-energy transfer line. The two BPMs are located in the middle of two double-bend achromats: SCL.01 is in the first achromat directly upstream of the collimator used for the energy acceptance measurement; SFEL01.03 is in the second achromat. The error bars show the standard error of the mean value of 17 consecutive measurements. 


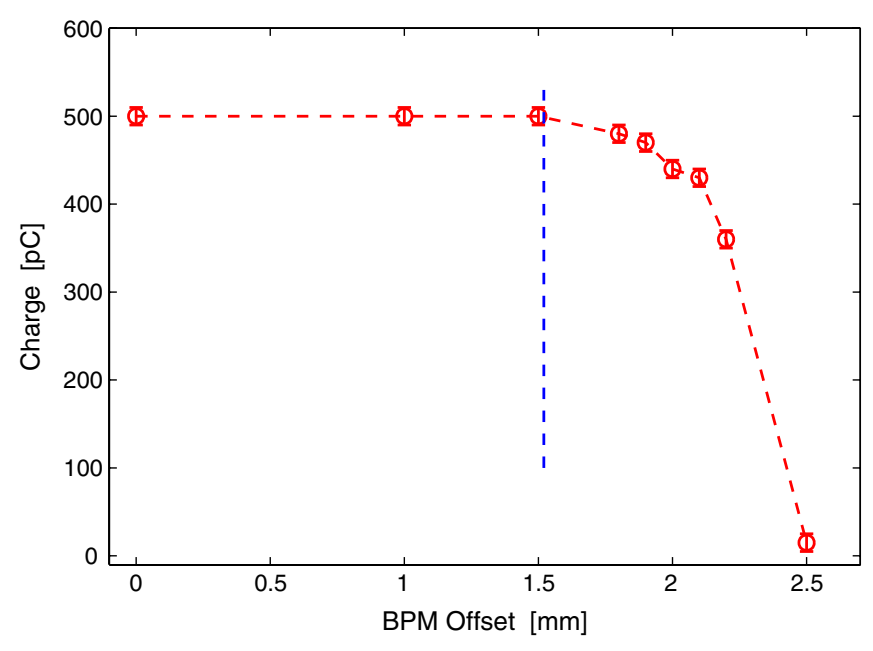

FIG. 6. The charge transmitted to the end of the FERMI transfer line vs the BPM offset. The energy collimator was set to the $2 \mathrm{~mm}$ half-gap hole.

of the FERMI linac) and measuring the resultant variation of the horizontal position at the BPM close to the collimator. Figure 5 shows a linear, least means-square fit applied to the experimental data. The fit provides a linear momentum dispersion of $\eta_{x}=(113 \pm 10) \mathrm{mm}$. The dispersion uncertainty is determined by the measurement reproducibility. The design value for the dispersion at the BPM is $114 \mathrm{~mm}$; for any practical purpose, the same value applies at the collimator location.

The collimator is then moved to the $2 \mathrm{~mm}$ radius hole, for which our transport calculations predict the nominal energy acceptance of $\pm 1.75 \%$. Figure 6 shows the charge measurement versus the BPM offset. The charge value first starts decreasing when the offset reaches $1.5 \mathrm{~mm}$. Taking into account the half beam size of $3 \sigma=$ $450 \mu \mathrm{m}$, we calculate an energy acceptance of $1.95 / 114=1.7 \% \pm 0.3 \%$.

\section{CONCLUSIONS}

A high brightness, $500 \mathrm{pC}$ electron beam was used for the measurement of the projected emittance growth induced by the transverse wakefield of the longitudinally tapered collimator in the FERMI@Elettra FEL. The measurement confirms that the beam quality is not degraded if the relative alignment between the beam and the collimator remains within a few hundred microns (e.g., $<1 \%$ emittance growth for $200 \mu \mathrm{m}$ misalignment). We determined a collimator kick factor of $2.20 \mathrm{~V} / \mathrm{pC} / \mathrm{mm}$, in good agreement with the theoretical expectation of $2.19 \mathrm{~V} / \mathrm{pC} / \mathrm{mm}$ and close to the value computed [10] for a similar dimension hole in FLASH for which the kick factor is $2.04 \mathrm{~V} / \mathrm{pC} / \mathrm{mm}$. The same type of collimator was adopted in FERMI for energy collimation. The measured energy acceptance is in excellent agreement with the theoretical value of $\pm 1.75 \%$. As a final remark, we notice that no halo particles were detected to the $4 \times 10^{-3}$ level of intensity measurements, for bunch charges up to $500 \mathrm{pC}$. We hope that these especially designed collimators and experimental findings will impact the future design and realization of compact collimation systems for single-pass, high brightness linacs.

[1] C. Adolphsen et al., Zeroth-Order Design Report for the Next Linear Collider (Stanford University, Stanford, 1996), Chap. 9, pp. 555-642.

[2] P. Tenenbaum, P. Emma, L. Keller, Y. Nosochkov, T. O. Raubenheimer, and M. Woodley, Report No. SLAC-PUB8934, 2001.

[3] T. Kamps, in Proceedings of the 26th Free Electron Laser Conference, Trieste, Italy, 2004 (Comitato Conferenze Elettra, Trieste, Italy, 2004), TUPOS02.

[4] I. Agapov, H. Burkhardt, D. Schulte, A. Latina, G. A. Blair, S. Malton, and J. Resta-López, Phys. Rev. ST Accel. Beams 12, 081001 (2009).

[5] K. Bane, C. Adolphsen, F. Decker, P. Emma, P. Krejcik, and F. Zimmermann, in Proceedings of the 16th Particle Accelerator Conference, Dallas, Texas, 1995 (IEEE, New York, 1995), RPA04.

[6] G. Stupakov, Part. Accel. 56, 83 (1996).

[7] R. J. Barlow, A. Bungau, and R. M. Jones, in Proceedings of the 22nd Particle Accelerator Conference, Albuquerque, New Mexico, 2007 (IEEE, New York, 2007), THPAN081.

[8] A. Bungau and R. Barlow, in Proceedings of the 22nd Particle Accelerator Conference, Albuquerque, New Mexico, 2007 (Ref. [7]), THPAN079.

[9] R. J. Barlow and A. M. Toader, in Proceedings of the 23rd Particle Accelerator Conference, Vancouver, Canada, 2009 (IEEE, Piscataway, NJ, 2009), TH5PFP084.

[10] A. Tsakanian, M. Dohlus, and I. Zagorodnov, Nucl. Instrum. Methods Phys. Res., Sect. A 659, 9 (2011).

[11] N. K. Watson et al., in Proceedings of the 10th European Particle Accelerator Conference, Edinburgh, United Kingdom, 2006 (EPS-AG, Edinburgh, Scotland, 2006), MOPLS066.

[12] I. Zagorodnov and K. Bane, in Proceedings of the 10th European Particle Accelerator Conference, Edinburgh, United Kingdom, 2006 (Ref. [11]), THPCH036.

[13] S. Molloy et al., in Proceedings of the 22nd Particle Accelerator Conference, Albuquerque, New Mexico, 2007 (Ref. [7]), FRPMS074.

[14] P. Tenenbaum, K. L. F. Bane, L. Eriksson, J. Irwin, R. K. Jobe, D. McCormick, C. K. Ng, T. O. Raubenheimer, M. C. Ross, G. Stupakov, D. Walz, D. Onoprienko, and I. Zagorodnov, Phys. Rev. ST Accel. Beams 10, 034401 (2007).

[15] S. Ferry and E. Karantzoulis, FERMI Internal Note Report No. FPRD-042, 2009.

[16] S. Ferry, C. Bontoiu, P. Craievich, S. Di Mitri, and E. Karantzoulis, in Proceedings of the 23rd Particle Accelerator Conference, Vancouver, Canada, 2009 (Ref. [9]), WE6RFP043. 
[17] FERMI@Elettra FEL Conceptual Design Report No. ST/ F-TN-07/12, 2007 [http://www.elettra.trieste.it/FERMI/ index.php?n=Main.CDRdocument].

[18] S. Di Mitri and M. Cornacchia, in Proceedings of the 32nd Free Electron Laser Conference, Malmo, Sweden, 2010, WEPB43.

[19] S. Di Mitri, Phys. Rev. ST Accel. Beams 13, 052801 (2010).
[20] M. G. Minty and F. Zimmermann, Report No. SLAC-R621, 2003.

[21] S. Di Mitri, E. M. Allaria, P. Craievich, W. Fawley, L. Giannessi, A. Lutman, G. Penco, S. Spampianti, and M. Trovo', Phys. Rev. ST Accel. Beams 15, 020701 (2012).

[22] M. Sands, Report No. SLAC-AP-85, 1991. 